\title{
Article \\ PSF Analysis of the Inverse Source and Scattering Problems for Strip Geometries
}

\author{
Ehsan Akbari Sekehravani (D), Giovanni Leone *(D) and Rocco Pierri (D) \\ Department of Engineering, University of Campania Luigi Vanvitelli, via Roma 29, I-81031 Aversa, Italy; \\ ehsan.akbarisekehravani@unicampania.it (E.A.S.); rocco.pierri@unicampania.it (R.P.) \\ * Correspondence: Giovanni.leone@unicampania.it
}

check for updates

Citation: Sekehravani, E.A.; Leone,

G.; Pierri, R. PSF Analysis of the

Inverse Source and Scattering

Problems for Strip Geometries.

Electronics 2021, 10, 754. https://

doi.org/10.3390/electronics10060754

Academic Editor:

Toshifumi Moriyama

Received: 13 February 2021

Accepted: 19 March 2021

Published: 22 March 2021

Publisher's Note: MDPI stays neutral with regard to jurisdictional claims in published maps and institutional affiliations.

Copyright: (c) 2021 by the authors. Licensee MDPI, Basel, Switzerland. This article is an open access article distributed under the terms and conditions of the Creative Commons Attribution (CC BY) license (https:// creativecommons.org/licenses/by/ $4.0 /)$.

\begin{abstract}
This paper is concerned with estimating the achievable resolution in the reconstruction of strip sources from the knowledge of its radiated field and strip objects from the knowledge of its scattered field. In particular, the study focuses on the evaluation of the point spread function (PSF), providing the reconstruction of a point-like unknown. Since this can be performed only numerically for most geometries, an approximate closed-form evaluation is introduced and compared with the exact one. Numerical results confirm the approximation accuracy, at least in the main lobe region of the PSF, which is the most important, as far as the discussion about resolution is concerned. The main results of the analysis concern the space invariance of the PSF of the considered geometries, which means that resolution is the same over the whole investigation domain, and the appreciation of its values for the inverse source and scattering problems.
\end{abstract}

Keywords: inverse source problem; inverse scattering problem; point spread function; resolution

\section{Introduction}

The study of inverse problems is widespread in science and engineering, and has been considered by mathematicians, statisticians, and engineers. It arises in a variety of significant applications in science [1] and industry [2]. The inverse source [3,4] and inverse scattering problem $[5,6]$ are two principle issues in electromagnetics problems.

The inverse source problem is a linear one that consists of reconstructing a current source from the knowledge of its radiated field in the frequency domain and some prior information. For instance, it requires determining an antenna's current distribution from its radiation pattern. Therefore, it is significant and has many applications, such as antenna design, and their testing and diagnostics.

Indeed, the inverse scattering problem is aimed at determining the dielectric features of an unknown scatterer, like its material, geometry (shape), and position, from information about its scattered fields under known excitation fields. Generally speaking, it turns out nonlinear unless an approximate scattering model is adopted as a small perturbation from a reference embedding medium, both homogenous (the classical Born approximation) or, even, inhomogenous (see [7]). The resulting problem has a wealth of applications in radar, subsurface, medical, acoustic, and synthetic aperture radar imaging, computer tomography, seismic, and electromagnetic geophysical exploration.

For instance, in optical communication theory, the transfer features of imaging systems are described as the point spread function (PSF) in the spatial domain. The computation of the output of the system corresponding to a given input in the spatial domain requires the convolution of the input with the PSF of the system, i.e., the system response to a pointlike object. In the frequency domain, the output is calculated by multiplying the input amplitude spectrum by the modulation transfer function of the system [8], i.e., the Fourier transform of the PSF. Since the imaging system determines the achievable image quality, the PSF is an appropriate tool for understanding the performance of the optical system. 
The PSF has been utilized to represent the impulse response of an optical imaging system to a point-like target object for imaging problems in optics [9]. In these circumstances, when the relevant imaging operator is the Fourier transform, a closed-form expression of the PSF is commonly available. In [10], the PSF has been used for earthquake source imaging.

A comparison [11] has been considered to analyze the resolution of geophysical images between the region of the data influence index, which is a nonlinear strategy, and the PSF, which is a linear approach.

The best-known consensus on image measurement in electrical impedance tomography uses PSFs, which are calculated over the whole domain as responses to a small circular anomaly moving around the entire domain [12]. A versatile criterion has been studied for estimating the quality of images in electrical impedance tomography. The PSF is calculated throughout the domain based on the scattering of energy as responses to a small anomaly spirally moved from the center to the boundary.

In [13], an analytical expression for the PSF of ultrasonic pulse-echo imaging has been addressed.

The imaging of a point-like scatterer in a medium under the Born approximation in the far-field by applying the PSF has been considered [14]; furthermore, the PSF has been used as a migration Green's function, and it has been shown, because of the image point positions and source-receiver configuration, to help identify migration artifacts.

The resolution issues in the inverse electromagnetic source problem have been considered, for instance, in [15], dealing with the problem of estimating depth-resolving power for the reconstruction of an electric current from Fresnel fields, or in [16], where it has shown how specific symmetry and support prior affect the number of degrees of freedom, the PSF and the Kolmogorov entropy.

The role of the source aspect ratio and the representation of different variables on the number of degrees of freedom (NDF), the PSF behavior, and the spectral content in the far-zone observation domain has been examined in [17] for different source geometries.

In [18], an analytical estimation for achievable resolution and linking it to configuration parameters has been addressed using the PSF for the magnetic and electric strip sources.

The role of the geometry in the inverse source reconstruction problem for far-field data has been analyzed in [19] for a semi-elliptic source when the observation domain is a semi-circumference. It has been shown that, for limited observation domains, the PSF is an angularly varying function, with different main lobes widths according to its maximum direction, even if we use different representation variables. The capacity to reconstruct point-like sources has also been considered in connection with the antenna array diagnostics problem.

In the inverse electromagnetic scattering problem, the study of the achievable resolution's depth has been considered to reconstruct the permittivity of a dielectric strip from Fresnel fields for multifrequency [20] or multistatic [21] data.

The PSF behavior analysis is also connected to the concept of the NDF of the problem, i.e., the number of independent pieces of information that can be reconstructed reliably by an imaging algorithm in the presence of uncertainties in data. Once the singular values decomposition (SVD) of the relevant operator is available, the NDF is provided by the number of its significant singular values and can be roughly assumed as the number of independent point-like sources that can be reconstructed reliably, so providing the maximum achievable resolution.

The SVD also allows the computation of the PSF by means of the right singular function [17], but, unfortunately, it can be performed in closed-form only in a few cases; for instance, the radiation of a linear current source [22] or a circumference one [23]. In most other instances, a numerical evaluation is required; in addition, the SVD and, then, the corresponding PSF depends on the geometry of the source domain. Therefore it would be beneficial to investigate whether an approximate, simpler, closed-form way of evaluating the PSF can be found. 
This paper is aimed at a PSF analysis for far-field observations to investigate the resolution in imaging. Furthermore, we deal with the issue of providing an approximation of the relevant PSFs for both the inverse source and the inverse scattering problems in the 2D scalar geometry. To this end, we consider strip geometries for a preliminary investigation as it is possible to find the NDF in closed-form. In this way, the connection between the NDF, the PSF, and the resolution in reconstructions can be highlighted clearly. In order to validate the analytical evaluation of the PSFs and their role in the resolution issue, we present some numerical simulations for each geometry.

The paper is organized as follows. Section 2 is devoted to the PSF analysis in the inverse source problem for the following cases: one strip, two strips, and cross-strip. We present the same analysis for the inverse scattering problem in Section 3. Finally, we give a brief summary and conclusions in Section 4.

\section{PSF Analysis in the Inverse Source Problem}

This section aims to provide some mathematical preliminaries and notations to be used in the following subsections. In particular, we recall the PSF definition, show its connection with the NDF and introduce its approximate evaluation. To this end, since the whole discussion is strictly linked to the radiation operator's singular values behavior, we investigate how to estimate them when the radiated field is collected over the whole observation domain in the far zone.

At first, we will address a general inverse source problem which is depicted in Figure 1. A scalar 2D source current $J$ radiates in a homogeneous medium with dielectric permittivity $\epsilon_{0}$ and magnetic permeability $\mu_{0}$, referred to as the source domain (SD). The observation direction of the radiated far-field is denoted by $\theta$.

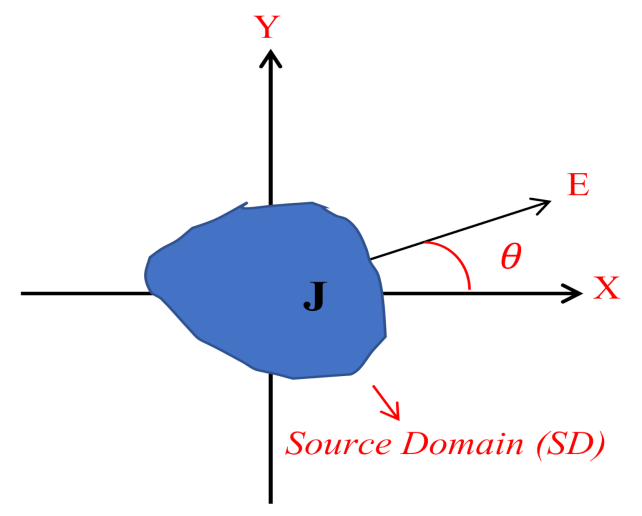

Figure 1. The general diagram of the inverse source problem.

Firstly, let us consider a 2D scalar current $J(x, y)$. At a single frequency, the electric far-field $E(\theta)$ is provided by the linear integral operator

$$
E(\theta)=\iint_{S D} J(x, y) e^{j \beta[x \cos \theta+y \sin \theta]} d x d y=\mathcal{L}(J(x, y))
$$

The wavenumber is denoted by $\beta=\omega \sqrt{\left(\epsilon_{0} \mu_{0}\right)}=2 \pi / \lambda$, where $\omega$ is the angular frequency and $\lambda$ is the wavelength.

We consider our problem in a complex Hilbert space $\mathcal{H}$. It consists of a linear mapping $\mathcal{L}: \mathcal{H}_{J} \rightarrow \mathcal{H}_{E}$ from a Hilbert space of source functions $\mathcal{H}_{J}$ to a Hilbert space of data $\mathcal{H}_{E}$ that is very general. The radiation operator mapping the vector space of current functions into one of the radiated fields is

$$
\mathcal{L}: J \in L^{2}([x, y] \in S D) \rightarrow E \in L^{2}([-\pi, \pi])
$$


where $J$ and $E$ are presumed to pertain to the set of square-integrable functions signified by $\mathcal{L}^{2}($.$) supported over the source domain [-\pi, \pi] \times[x, y]$ and the observation domain $[-\pi, \pi]$, respectively. Now, since the $\mathcal{L}$ operator is linear and compact, the SVD can be defined for each source geometry and consists of the triple $\left\{v_{n}(x, y), \sigma_{n}, u_{n}(\theta)\right\}$ [19] where $u_{n}$ is the $n$-th right singular function, $\sigma_{n}$ is the $n$-th singular value and $v_{n}$ is the $n$-th left singular function, respectively.

In particular, the $u_{n}$ functions provide the class of achievable far-field functions and the $v_{n}$ functions, the set of possible currents. Moreover, the behavior of the singular values $\sigma_{n}$ provides the number of independent currents/patterns that the source can radiate. Thus, since they decay exponentially fast, the NDF of the source can be introduced.

The adjoint mapping of $\mathcal{L}, \mathcal{L}^{+}: \mathcal{H}_{E} \rightarrow \mathcal{H}_{J}$,

$$
\mathcal{L}^{+}: E \in L^{2}([-\pi, \pi]) \rightarrow J \in L^{2}([x, y] \in S D)
$$

is defined as

$$
\langle E \mid \mathcal{L} J\rangle_{\mathcal{H}_{E}}=\left\langle\mathcal{L}^{+} E \mid J\right\rangle_{\mathcal{H}_{J}}
$$

where the standard definitions of the inner products in the two spaces are assumed. Therefore, because of (1),

$$
\mathcal{L}^{+}(E)=\int_{-\pi}^{\pi} E(\theta) e^{-j \beta\left[x^{\prime} \cos \theta+y^{\prime} \sin \theta\right]} d \theta
$$

Next, from the spectral theorem for compact self-adjoint operators applied to the cascade $\mathcal{L}^{+} \mathcal{L}$, we can compute

$\mathcal{L}^{+} \mathcal{L}(J(x, y))=\int_{-\pi}^{\pi} e^{-j \beta\left[x^{\prime} \cos \theta+y^{\prime} \sin \theta\right]}\left[\iint_{S D} J(x, y) e^{j \beta[x \cos \theta+y \sin \theta]} d x d y\right] d \theta=$

whose kernel is

$$
K\left(x-x^{\prime}, y-y^{\prime}\right)=\int_{-\pi}^{\pi} e^{j \beta\left[\left(x-x^{\prime}\right) \cos \theta+\left(y-y^{\prime}\right) \sin \theta\right]} d \theta=2 \pi J_{0}\left(\beta \sqrt{\left(x-x^{\prime}\right)^{2}+\left(y-y^{\prime}\right)^{2}}\right)
$$

where $J_{0}$ is the zeroth-order Bessel function of the first kind.

Finally, substituting Equation (7) to (6), it reads as

$$
\mathcal{L}^{+} \mathcal{L}(J(x, y))=\iint_{S D} J(x, y)\left[2 \pi J_{0}\left(\beta \sqrt{\left(x-x^{\prime}\right)^{2}+\left(y-y^{\prime}\right)^{2}}\right)\right] d x d y
$$

Now, in order to assess the performance of the reconstruction algorithm, the PSF is considered. In the inverse source problem, the PSF analysis is performed to realize how the source geometry and the observation domain may affect the resolution. Here, we intend to analyze only the influence of the source geometry. The aim is to get an analytical estimation for the achievable resolution and connect it to the geometrical parameters. To this end, we start to consider the PSF for a general 2D source and then apply it to the other simpler source geometries.

The PSF is the observed current distribution in the SD for a point source located at $x_{0}$ and $y_{0}$. From the mathematical point of view, the PSF in the source domain is defined as the impulsive response of the system provided by the cascade operator $\mathcal{L}^{-1} \mathcal{L}$, as

$$
\operatorname{PSF}\left(x, x_{0}, y_{1} y_{0}\right)=\left(\mathcal{L}^{-1} \mathcal{L}\right) \delta\left(x-x_{0}\right) \delta\left(y-y_{0}\right)
$$

where $\mathcal{L}^{-1}$ is the regularized inverse operator of $\mathcal{L}$ and $\delta$ is the Dirac delta-function.

Thus, since the minimum-norm solution of the inverse source problem is a projection of the actual source onto the singular function $v_{n}$ having non-zero singular values, the 
PSF for the inverse source problem is given by the completeness relation truncated to the singular functions having non-zero singular values; therefore, in general, the PSF is dependent on the number of retained singular values, which, in turn, is connected to the noise level. Then if a truncated SVD (TSVD) [24] inversion scheme is adopted to implement the regularized inversion step, Equation (9) can be written explicitly as

$$
\operatorname{PSF}\left(x, x_{0}, y_{1} y_{0}\right)=\sum_{n=1}^{N} v_{n}(x, y) v_{n}^{*}\left(x_{0}, y_{0}\right)
$$

where $*$ means conjunction operation and $N$ is a truncation index, which can be estimated as the knee of the singular values curve. Therefore, the $N$ value can be selected in terms of the NDF.

Equation (10) requires the knowledge of the singular functions, which can be computed in closed form only for limited instances of source geometries. On the other hand, if the adjoint operator may approximate the inverse operator in Equation (9), it can be considered to build a good approximation of the PSF $[15,25]$. This occurs when the singular values of the radiation operator exhibit a nearly constant behavior before the knee of their curve. Consequently, by replacing the adjoint operator instead of the inversion operator in (9), we define the approximated $\widetilde{P S F}$ by

$$
\widetilde{\operatorname{PSF}}\left(x, x_{0}, y, y_{0}\right)=\left(\mathcal{L}^{+} \mathcal{L}\right) \delta\left(x-x_{0}, y-y_{0}\right)
$$

Therefore, Equation (8) provides the explicit analytical evaluation of (11) for the 2D geometry under consideration.

We now turn to provide a comparison of the exact and approximated results of PSFs for some different geometries composed of a collection of linear sources, i.e., the one strip case first, the two parallel strip one next, and finally the cross-strip one, in Sections 2.1-2.3, respectively.

\subsection{One Strip Case}

In this subsection, we address the case of the electric field radiated by a z-invariant electric current $J$ strip source, supported over the interval $I=[-a, a]$ of the x-axis. So, the problem is scalar, and the radiated electric field has only the $z$ component. Figure 2 shows the geometry of the problem. The aim is to compare the actual PSF and the approximated $\widetilde{P S F}$ moreover, to verify the space invariance by numerical simulations.

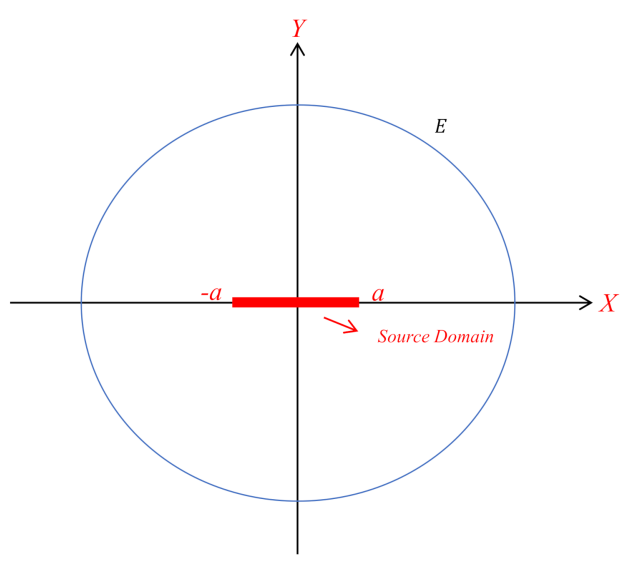

Figure 2. The geometry of the problem.

We define the relevant operator for one strip case as

$$
E(\theta)=\int_{-a}^{a} J(x) e^{j \beta x \cos \theta} d x=\mathcal{L}_{1 s}(J(x))
$$


where $\mathcal{L}_{1 s}$ is a linear operator, and the adjoint operator $\mathcal{L}_{1 s}^{+}$of $\mathcal{L}_{1 s}$ is obtained by

$$
\mathcal{L}_{1 s}^{+}=\int_{-\pi}^{\pi} E(\theta) e^{-j \beta x \cos \theta} d \theta
$$

By considering the approximation, as mentioned above, the PSF and $\widetilde{P S F}$ can be easily derived by replacing $\mathcal{L}_{1 s}$ and $\mathcal{L}_{1 s}^{+}$instead of $\mathcal{L}$ and $\mathcal{L}^{+}$in Equations (9) and (11), respectively. The approximated $\widetilde{P S F}$ is given by

$$
\widetilde{P S F}=2 \pi J_{0}\left(\beta\left|x-x_{0}\right|\right)
$$

Since for a linear source, the kernel of the operator $\mathcal{L}^{+} \mathcal{L}$ has a convolutional form, the relevant $\widetilde{P S F}$ exhibits an invariant behavior along with all the source domains. Hereafter, we provide some numerical examples to assess the accuracy of (14) again with the exact PSF and, hence, to appreciate the resolution. To this end, we neglect all the side lobes of the $\widetilde{P S F}$ as it is enough to consider only its main lobe to outline the focusing properties. Moreover, all PSFs are normalized to 1 . Figure 3 shows the comparison of the PSF and $\widetilde{P S F}$ for three different source points located at the $x_{0}=-2 \lambda, 0$, and $\lambda$. It can be recognized that the resolution remains unchanged as the position of $x_{0}$ varies.

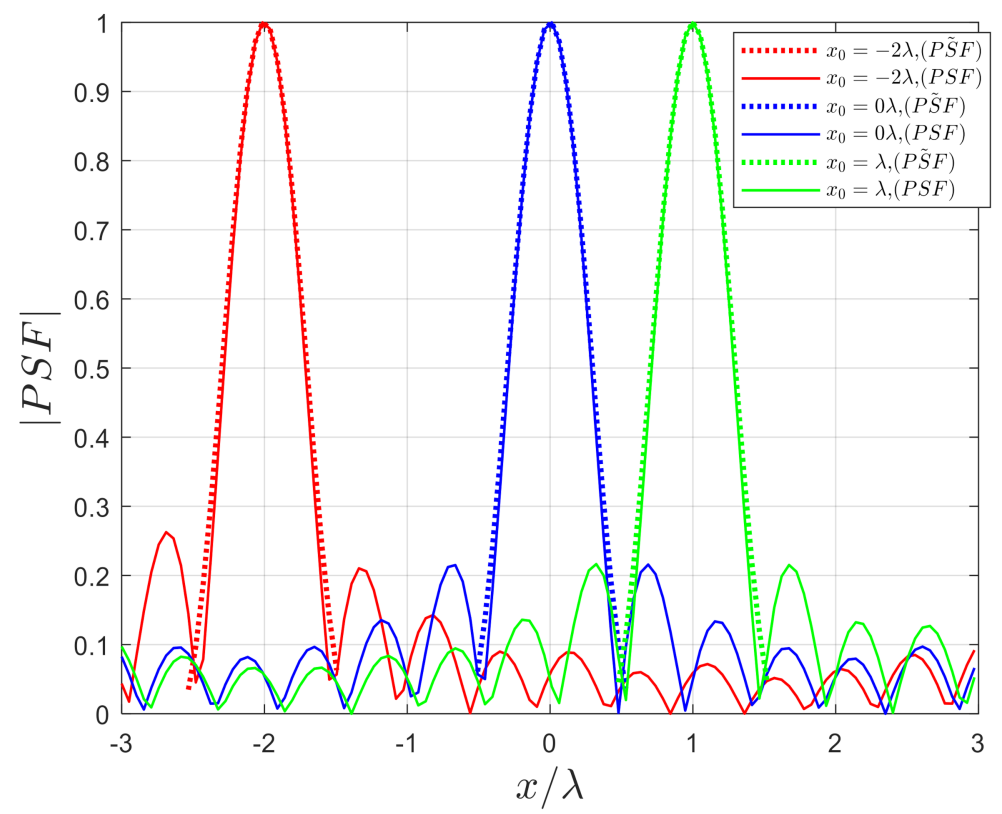

Figure 3. Comparison of normalized actual point spread function $(P S F)$ and approximated $\widetilde{P S F}$ for $x_{0}=-2 \lambda, 0, \lambda$, with $a=3 \lambda$.

Figure 4 confirms the results of the comparison of Equation (14) and the actual PSF, as the main lobes overlap very well. It means that (14) is very accurate in predicting the achievable resolution. 


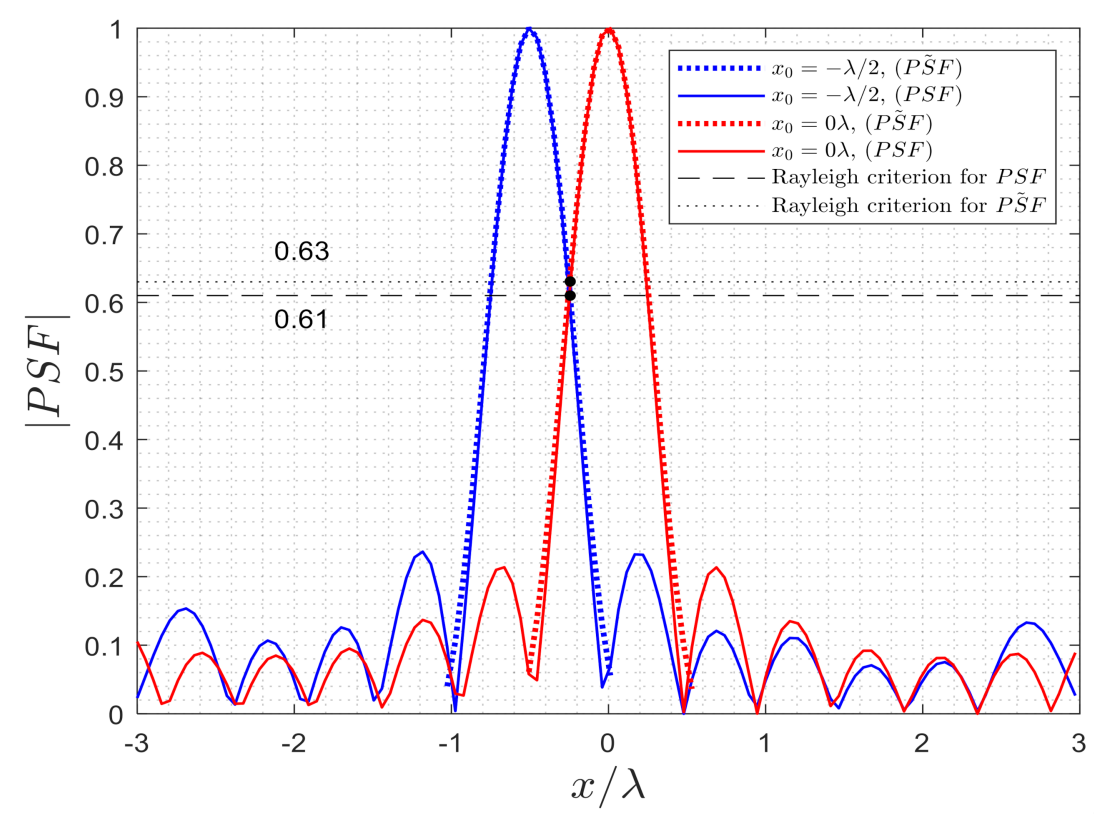

Figure 4. The Rayleigh criterion for PSF and $\widetilde{P S F}$.

In particular, while the PSF maximum value may depend on the source point, its width does not change and is independent of the position of the point source. Consequently, since the resolution in reconstructions of point-like sources is limited by the width of the PSF, it can be concluded that it is the same over the whole SD.

Resolution limits in source reconstructions are commonly introduced by means of the Rayleigh criterion as the minimum detectable separation between two close point-like sources. It can be appreciated by referring to the degree of overlap of the main lobes of the corresponding PSFs pattern. Two-point sources are generally regarded as resolved if the first maximum of one PSF falls into the first minimum of the other PSF, but there is no standard definition. However, the space invariance of the PSF can lead to introducing a quantitive assessment. In fact, as is well known [22], the NDF of this linear source is $[4 a / \lambda]$, where $[\cdot]$ stands for the nearest integer, and one can assume that the same number of point-like sources can be reconstructed.

Since the PSF is space invariant, which means that the reconstruction of a pointlike source is independent of its location, these sources can be uniformly distributed along with the $2 a$ long SD, leading to the typical $\lambda / 2$ resolution limit. Then, in order to introduce a quantitive measure for the Rayleigh criterion, we can consider two-point sources located in $x_{0}=-\lambda / 2$ and $x_{0}=0 \lambda$ and consider the level of the intersection point of the corresponding PSFs (Figure 4). As can be observed, the intersection point of $\widetilde{P S F}$ (dotted black line) is approximately close to the intersection point of PSF (dashed black line) so it can be used for sampling to find the optimal position and the distance between two samples in the source and observation domain.

\subsection{Two Strips Case}

The same analysis as the previous subsection is here extended to address the case of two parallel strips along the x-axis. Let us consider two strips that are located at $I_{1}=[-a, a]$ and $I_{2}=[-a, a]$, respectively, and $d=2 b$ is the distance between them (see Figure 5). This section aims to estimate the resolution and to compare the PSF and $\widetilde{P S F}$; moreover, we will show the influence of PSF of strip 1 on the other strip. 


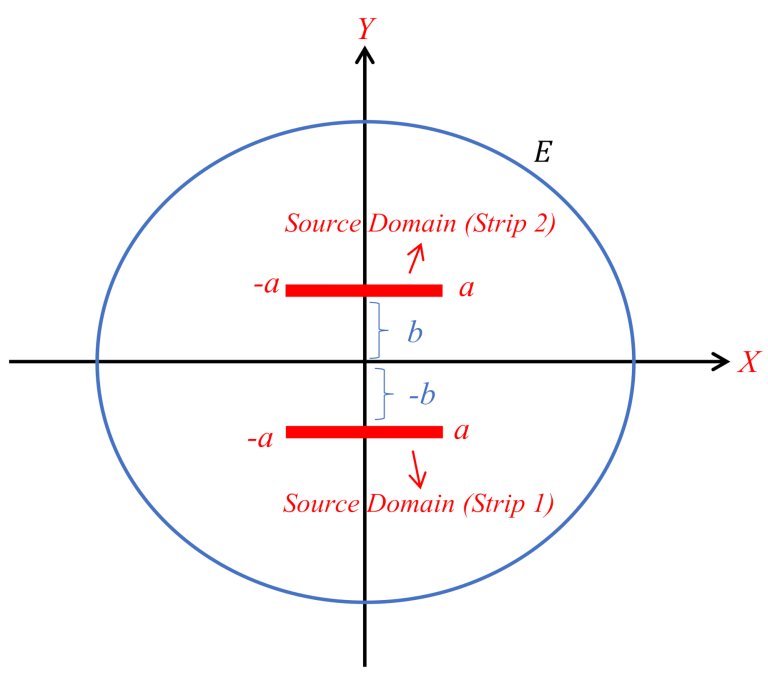

Figure 5. The geometry of two strip sources.

We define the relevant operator

$$
E_{T}(\theta)=\left[\begin{array}{lll}
e^{-j \beta b \sin \theta} & \mathcal{L}_{1 s} & e^{j \beta b \sin \theta} \mathcal{L}_{1 s}
\end{array}\right]\left[\begin{array}{c}
J_{1}\left(x_{1}\right) \\
J_{2}\left(x_{2}\right)
\end{array}\right]=\mathcal{L}_{2 s} J
$$

The adjoint operator $\mathcal{L}_{2 s}^{+}$of $\mathcal{L}_{2 s}$ is given by

$$
\mathcal{L}_{2 s}^{+}=\left[\begin{array}{cc}
\mathcal{L}_{1 s}^{+} & e^{j \beta b \sin \theta} \\
\mathcal{L}_{1 s}^{+} & e^{-j \beta b \sin \theta}
\end{array}\right] E_{T}
$$

Some numerical simulations are presented to confirm the analytical definition of the PSFs. Let us consider the geometry shown in Figure 5, where $a=3 \lambda$ and $b=\lambda$. The actual PSF of strip 1 and its influence on strip 2 are shown in Figure 6 for three different point sources, located at $x_{0}=-2 \lambda, 0$, and $\lambda$. As can be seen, by changing the point source's position, the resolution does not change. Furthermore, the result shows that the PSF result of strip 1 cannot affect strip 2 .
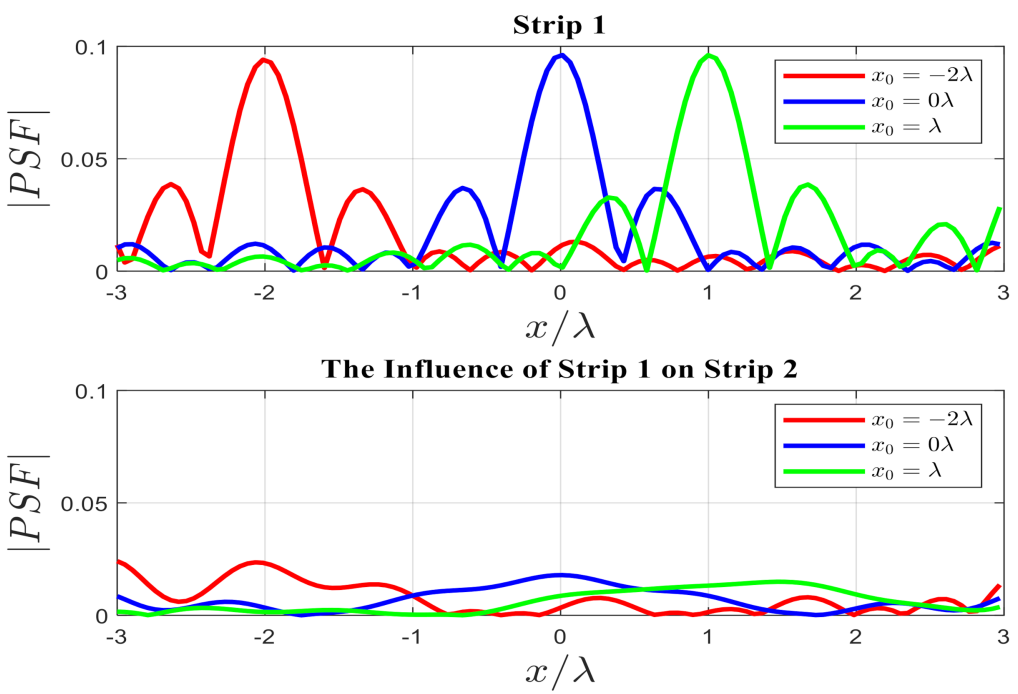

Figure 6. The influence of the PSF of strip 1 on strip 2.

The same simulation as in the previous subsection is here provided to compare the results of the relevant PSF and the $\widetilde{P S F}$ for different positions of $x_{0}$. As demonstrated in Figure 7 , the main lobes of the approximated $\widetilde{P S F}$ (dotted lines) and the actual PSF (solid 
lines) are approximately overlapped. Again, we observed that the width of PSFs does not vary when the position of the point source changes.

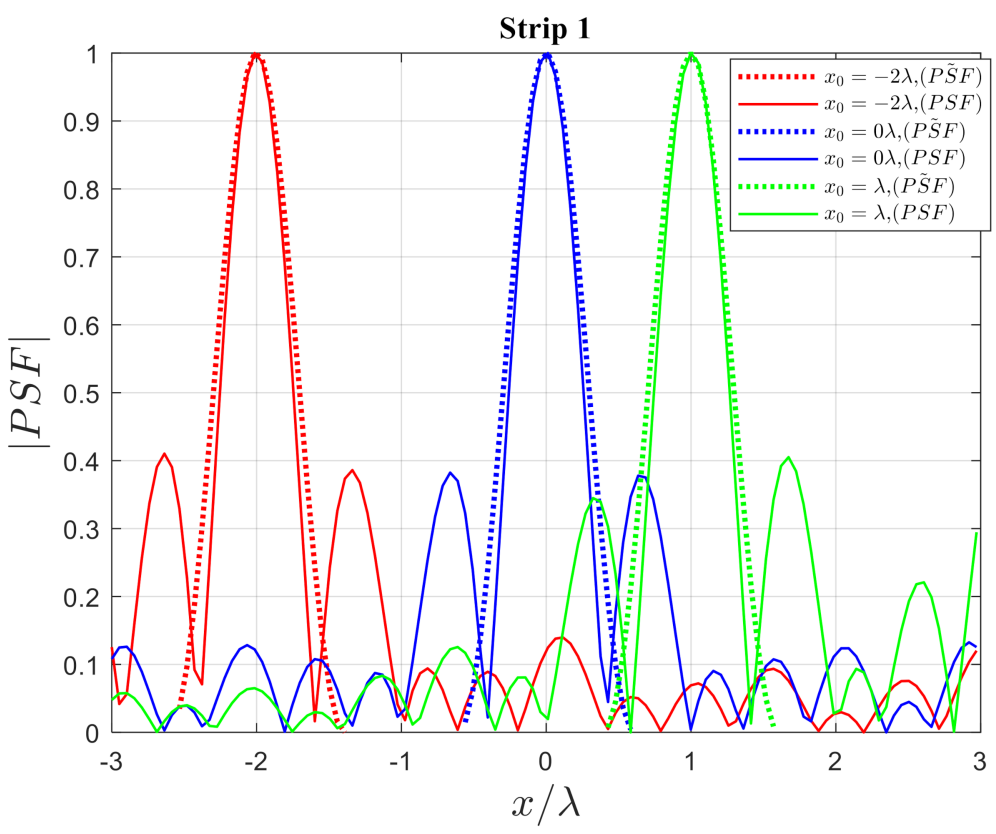

Figure 7. Comparison of normalized actual PSF and approximated $\widetilde{P S F}$ for $x_{0}=-2 \lambda, 0, \lambda$.

In order to relate the above-discussed concepts and numerical simulations to a practical application concerning the resolution issue in inverse source problems, we provide a numerical example concerning the diagnostics of faulty elements within an array antenna. It is composed of two sub-arrays of isotropic elements: one is located along the left part of strip 1 and is composed of 7 elements spaced at a uniform $\lambda / 2$ step, while the other one is located along the right part of strip 2 and is composed of 16 elements spaced at a uniform $\lambda / 5$ step. The elements of each sub-array are excited according to (different) constant values, chosen in such a way that each sub-array radiated the same power. Then, we suppose that two elements are inactive in each sub-array, as plotted in Figure 8, and we require them to be located by inversion of (15) by the truncated singular values decomposition (TSVD) algorithm. Here, since the array source is considered a particular case of a continuous source function, we can introduce a threshold value (dotted line) for the reconstructed amplitude of the current at half of its maximum values and decide that an element is off whenever the current value at its location is lower than the threshold. It can be expected that when the distance between two elements is lower than the $\lambda / 2$ resolution limit, it is not possible to reconstruct them reliably. Thus (see Figure 8), the diagnostics procedure is reliable for the sub-array of strip 1 , as the presence of defective elements can be unambiguously detected. In contrast, it is impossible to reconstruct every set of the excitation coefficients of the sub-array of strip 2. Since an antenna testing engineer must be confident that every collection of the excitation coefficients of the array must be retrievable, the reliability of the inversion algorithm can be significant and useful. 


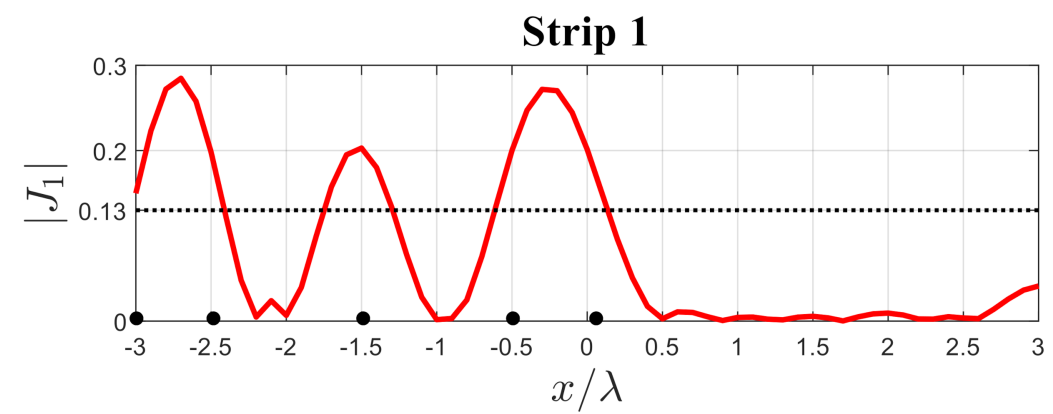

Strip 2

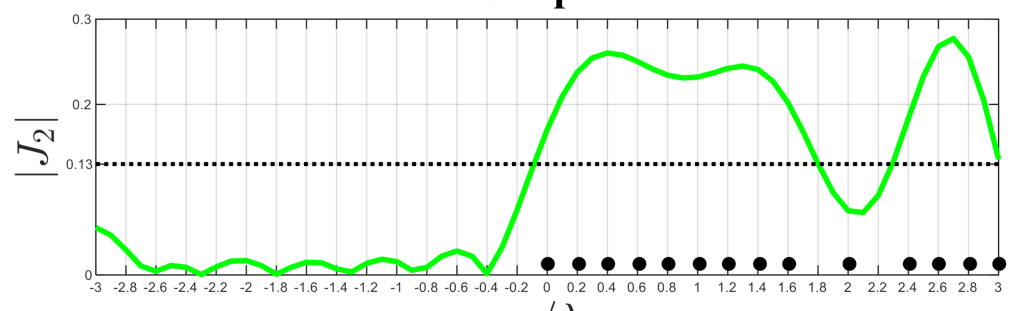

$x / \lambda$

Figure 8. The reconstruction (red and green lines) of the amplitude of the currents along each strip. The black dots indicate the position of the active array elements.

\subsection{Cross-Strip Case}

The subsection deals with the cross-strip geometry made of two identical $2 a$ long strips, with one along the x-axis and one along the y-axis (see Figure 9). They are considered to represent a connected source geometry.

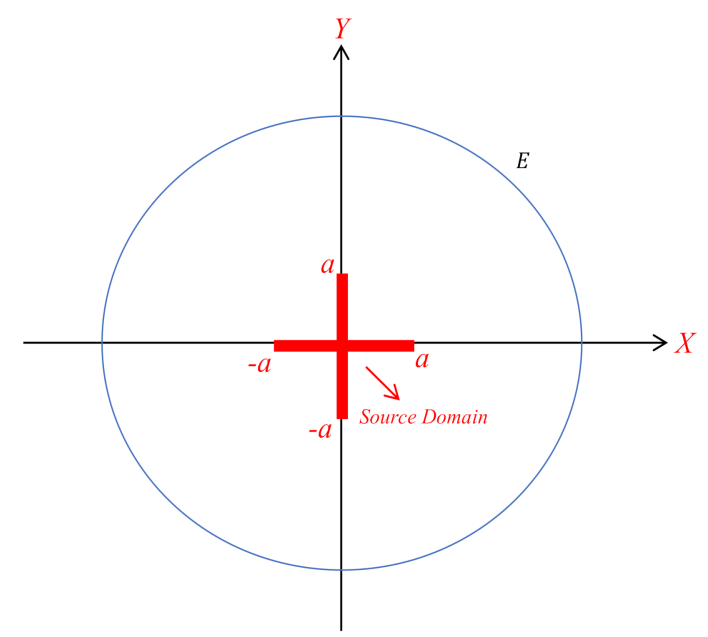

Figure 9. The geometry of the cross-strip.

Recalling Equation (12), the radiated far-field by the horizontal strip and the vertical one are defined, respectively, by

$$
E_{h}(\theta)=\int_{-a}^{a} J_{x}(x) e^{j \beta x \cos \theta} d x=\mathcal{L}_{x}\left(J_{x}(x)\right)
$$

and

$$
E_{v}(\theta)=\int_{-a}^{a} J_{y}(y) e^{j \beta y \sin \theta} d y=\mathcal{L}_{y}\left(J_{y}(y)\right)
$$

We can consider the operator (19)

$$
E(\theta)=\left[\begin{array}{ll}
E_{h}(\theta) & E_{v}(\theta)
\end{array}\right]=\left[\begin{array}{ll}
\mathcal{L}_{x} & \mathcal{L}_{y}
\end{array}\right]\left[\begin{array}{c}
J_{x}(x) \\
J_{y}(y)
\end{array}\right]=\mathcal{L}_{x y} J
$$


where the PSF and the $\widetilde{P S F}$ are defined as in the previous subsections.

Figure 10 shows the influence of the PSF of the horizontal strip on the vertical strip. As shown, the PSF of $x_{0}=0 \lambda$ can equally affect both strips. On the other hand, the PSF of the other point scatterer cannot affect the vertical strip. We observe again that by changing the position of the point scatterer, the width of the PSF remains constant; however, the amplitude varies.
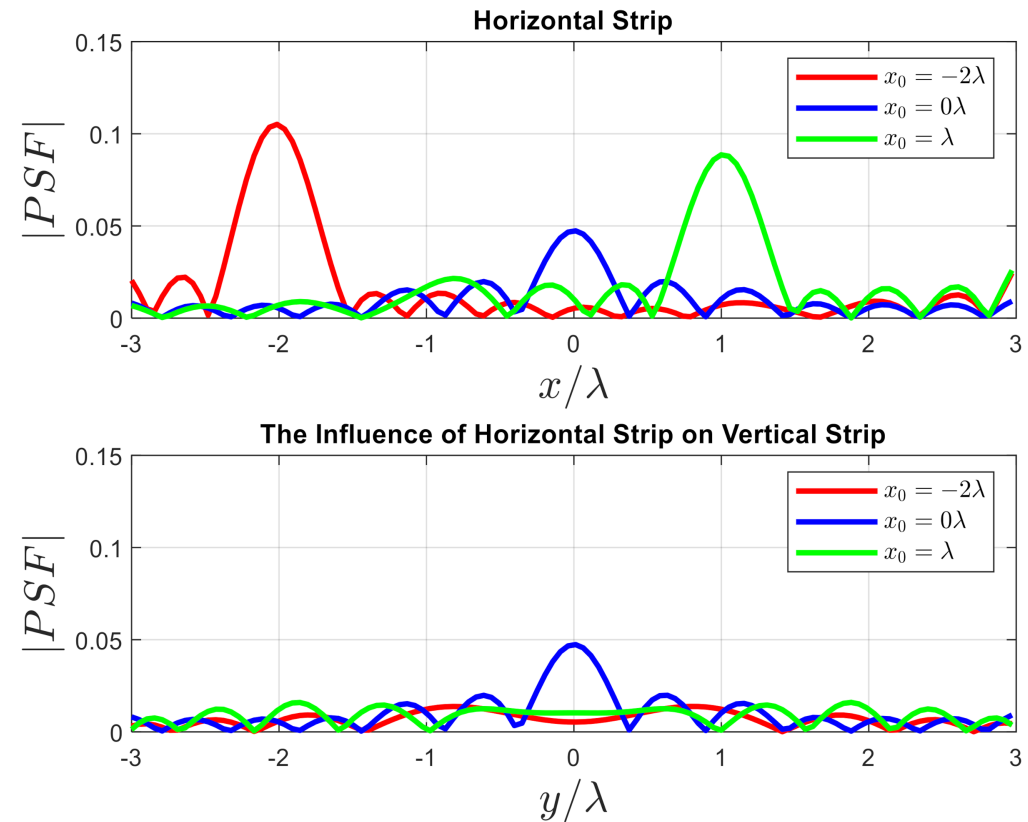

Figure 10. The influence of the PSF of the horizontal strip on the vertical strip, with $a=3 \lambda$.

Figure 11 illustrates a comparison between the relevant PSF and the $\widetilde{P S F}$ for different positions of $x_{0}$. The main lobes of the $\widetilde{P S F}$ (see dotted curves) are approximately overlapped with the actual one, which means the $\widetilde{P S F}$ is very accurate in predicting the achievable resolution.

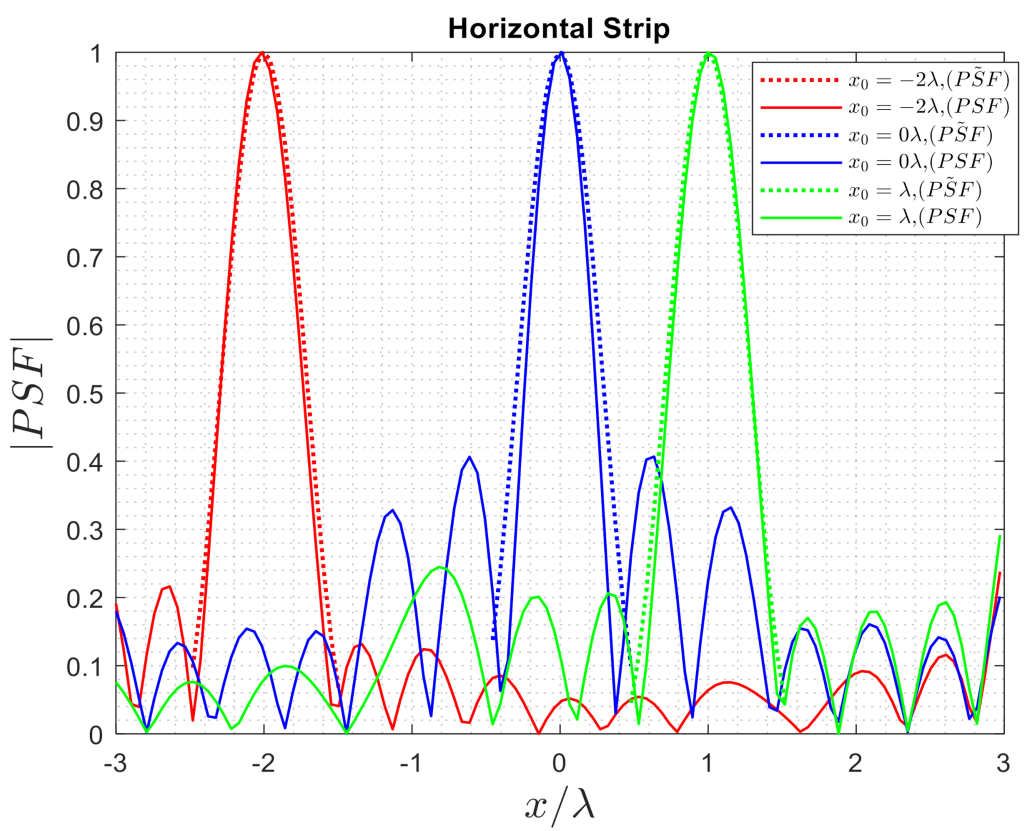

Figure 11. Comparison of normalized actual PSF and approximated $\widetilde{P S F}$ for $x_{0}=-2 \lambda, 0, \lambda$.

As can be observed in Figure 12, the intersection point of $\widetilde{P S F}$ (dotted black line) is approximately close to the intersection point of PSF (dashed black line). 


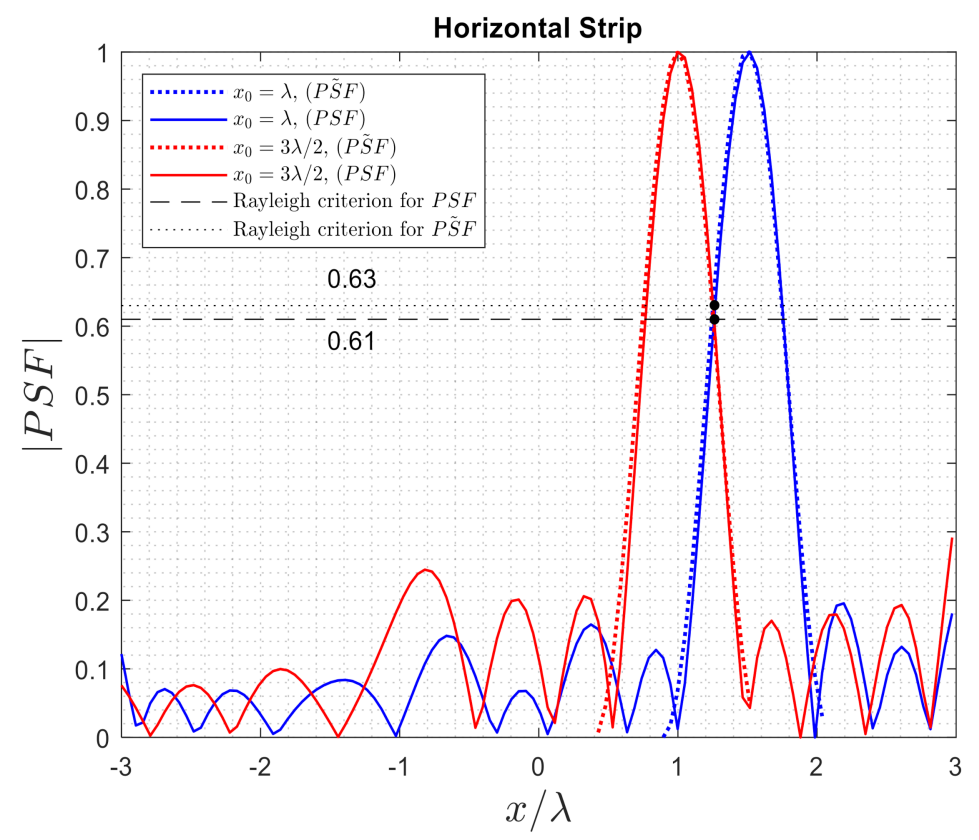

Figure 12. The Rayleigh criterion for PSF and $\widetilde{P S F}$.

\section{PSF Analysis of the Inverse Scattering Problem}

In this section, firstly, we define and formulate general inverse scattering problems (Figure 13). Secondly, we define the actual PSF and approximated $\widetilde{P S F}$ for 2D geometry.

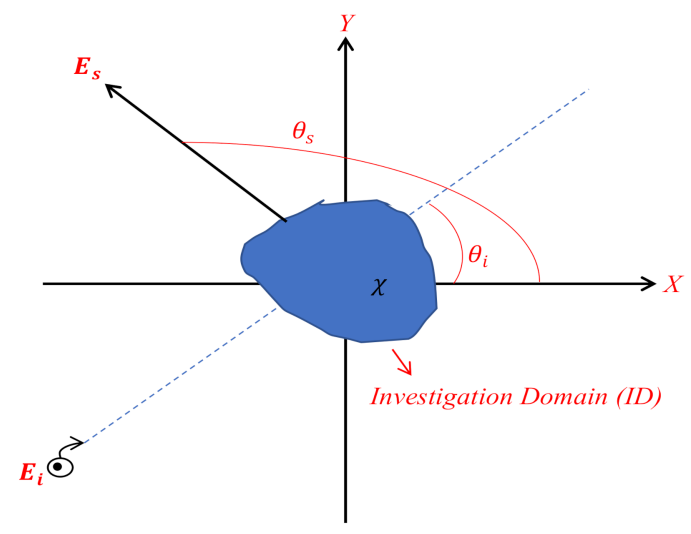

Figure 13. The general inverse scattering problems.

Let us consider that an unknown scatterer is located in a domain referred to as the investigation domain (ID), and the incident plane wave $E_{i}$, propagating along the $\theta_{i}$ direction illuminates it; then, for each angle of illumination, the scattered fields $E_{s}$ are observed at the $\theta_{s}$ observation angles in the scatterer's far zone.

Hence, the scattered far-field under the Born approximation can be recast as

$$
E_{S}\left(\theta_{s}\right)=\iint_{I D} \chi(x, y) E_{i}\left(x, y, \theta_{i}\right) e^{j \beta\left(x \cos \theta_{s}+y \sin \theta_{s}\right)} d x d y
$$

where $\chi(x, y)$ and $E_{i}\left(\theta_{i}\right)$ are the contrast function and the incident plane wave, respectively. The scattering object has a relative permittivity of $\epsilon_{s}(x, y)$, so that $\chi(x, y)=1-\left(\epsilon_{s}(x, y)\right) \epsilon_{0}$, and is located in a homogeneous background, which has the free space permittivity $\epsilon_{0}$. The incident plane wave is provided by

$$
E_{i}\left(\theta_{i}\right)=e^{-j \beta\left(x \cos \theta_{i}+y \sin \theta_{i}\right)}
$$


Now, the substitution of Equation (21) in (20) gives

$$
E_{S}\left(\theta_{s}, \theta_{i}\right)=\iint_{I D} \chi(x, y) e^{j \beta\left[x\left(\cos \theta_{s}-\cos \theta_{i}\right)+y\left(\sin \theta_{s}-\sin \theta_{i}\right)\right]} d x d y=\mathcal{T}(\chi(x, y))
$$

where $\mathcal{T}$ is the linear operator for the multi-view and single frequency scattering configurations of our present interest. As in Section 2, since $\mathcal{T}$ is a linear and compact operator, the SVD can be applied to express the PSF in terms of the singular functions of the operator.

In [26], we studied the behavior of the singular values of the considered operator, evaluated its NDF, investigated how many plane waves are needed to achieve the NDF, and introduced the optimal directions of the plane waves for the same geometries that we will analyze in this section.

The adjoint operator of (22) is

$$
\mathcal{T}^{+}\left(E_{S}\right)=\int_{-\pi}^{\pi} \int_{S}\left(\theta_{S}, \theta_{i}\right) e^{-j \beta\left[x \prime\left(\cos \theta_{s}-\cos \theta_{i}\right)+y \prime\left(\sin \theta_{s}-\sin \theta_{i}\right)\right]} d \theta_{S} d \theta_{i}
$$

and then, from the spectral theorem for compact self-adjoint operators applied to $\mathcal{T}^{+} \mathcal{T}$, it follows that

$$
\begin{aligned}
& \mathcal{T}^{+} \mathcal{T}(\chi(x, y))= \\
& \iint_{-\pi}^{\pi} e^{-j \beta\left[x^{\prime}\left(\cos \theta_{s}-\cos \theta_{i}\right)+y^{\prime}\left(\sin \theta_{s}-\sin \theta_{i}\right)\right]}\left[\iint_{I D} \chi(x, y) e^{j \beta\left[x\left(\cos \theta_{s}-\cos \theta_{i}\right)+y\left(\sin \theta_{s}-\sin \theta_{i}\right)\right]} d x d y\right] d \theta_{s} d \theta_{i}= \\
& \iint_{I D} \chi(x, y)\left[\iint_{-\pi}^{\pi} e^{j \beta\left[\left(x-x^{\prime}\right)\left(\cos \theta_{s}-\cos \theta_{i}\right)+\left(y-y^{\prime}\right)\left(\sin \theta_{s}-\sin \theta_{i}\right)\right]} d \theta_{s} d \theta_{i}\right] d x d y
\end{aligned}
$$

where the kernel of the Equation (24) is

$$
\begin{aligned}
& k\left(x-x \prime, y-y^{\prime}\right)=\iint_{-\pi}^{\pi} e^{j \beta\left[(x-x \prime)\left(\cos \theta_{s}-\cos \theta_{i}\right)+(y-y \prime)\left(\sin \theta_{s}-\sin \theta_{i}\right)\right]} d \theta_{s} d \theta_{i}= \\
& \quad \int_{-\pi}^{\pi} e^{j \beta\left[\left(x-x^{\prime}\right) \cos \theta_{s}+\left(y-y^{\prime}\right) \sin \theta_{s}\right]} d \theta_{s} \int_{-\pi}^{\pi} e^{-j \beta\left[\left(x-x^{\prime}\right) \cos \theta_{i}+\left(y-y^{\prime}\right) \sin \theta_{i}\right]} d \theta_{i}=\left(2 \pi J_{0}\left(\beta \sqrt{(x-x \prime)^{2}+\left(y-y^{\prime}\right)^{2}}\right)\right)^{2}
\end{aligned}
$$

is provided by the square of the Bessel function of the first kind and zeroth order. Finally, substituting Equation (25) to (24) results as

$$
\mathcal{T}^{+} \mathcal{T}(\chi(x, y))=\iint_{I D} \chi(x, y)\left(2 \pi J_{0}\left(\beta \sqrt{(x-x)^{2}+\left(y-y^{\prime}\right)^{2}}\right)\right)^{2} d x d y
$$

In the inverse scattering problem, the PSF analysis is performed to understand how the scatterer geometry and the observation domain may affect the resolution. Here we consider only the influence of scatterer geometry on the resolution. The PSF represents the reconstruction of a point scatterer. The adjoint operator $\mathcal{T}^{+}$can be used to approximate the inverse operator $\mathcal{T}^{-1}$ as in Section 2, so that an approximate $\widetilde{P S F}$ can be introduced as well. In the following, we compare both PSFs for the same geometries that are considered in the previous section: Section 3.1 provides the single strip case, whereas the two parallel strips and cross-strip are considered in Sections 3.2 and 3.3, respectively.

\subsection{One Strip Case}

Let us consider that one strip is located on the x-axis, as shown in Figure 14. First, we analyze the theoretical of the problem to estimate the resolution and then simulate it numerically. 


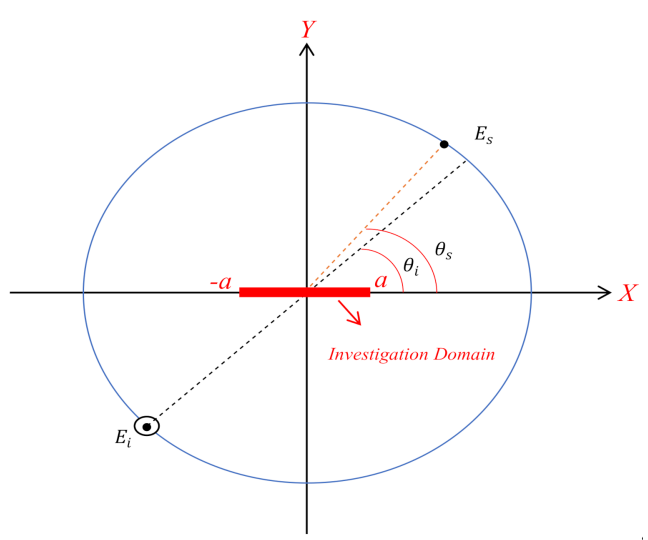

Figure 14. The geometry of the problem.

The scattered far-field of one strip is derived from Equation (22)

$$
E_{\mathcal{S}}\left(\theta_{\mathcal{S}}, \theta_{i}\right)=\int_{-a}^{a} \chi(x) e^{j \beta x\left(\cos \theta_{s}-\cos \theta_{i}\right)} d x=\mathcal{T}_{1 s}(\chi(x))
$$

The adjoint operator $\mathcal{T}_{1 s}^{+}$of the operator $\mathcal{T}_{1 s}$ is

$$
\mathcal{T}_{1 s}^{+}=\int_{-\pi}^{\pi} \int_{-} E_{s}\left(\theta_{s}, \theta_{i}\right) e^{-j \beta x^{\prime}\left(\cos \theta_{s}-\cos \theta_{i}\right)} d \theta_{s} d \theta_{i}
$$

The PSFs were previously defined for the 2D case in Section 2 so that it can be easily derived from Equations (9) and (11) for one strip case by replacing $\mathcal{T}_{1 s}^{-1}$ and $\mathcal{T}_{1 s}^{+}$instead of $\mathcal{L}^{-1}$ and $\mathcal{L}^{+}$, respectively. The approximated $\widetilde{P S F}$ for one strip is obtained by

$$
\widetilde{P S F}=\left(2 \pi J_{0}\left(\beta\left|x-x_{0}\right|\right)\right)^{2}
$$

The analytical PSF is verified using a numerical implementation of the imaging process when it can be assumed that one or more-point scatterers are located at an arbitrary position of the investigation domain. The PSFs for three-point scatterers located at $x_{0}=$ $-2 \lambda, 0$, and $\lambda$ are shown in Figure 15. Since the PSF is a function only of $x-x_{0}$ we have arbitrarily selected the point scatterer locations along the x-axis. It can be observed that the resolution remains unchanged as the position of $x_{o}$ varies. All point-like scatterers can be imaged in the same way, independent of their positions.

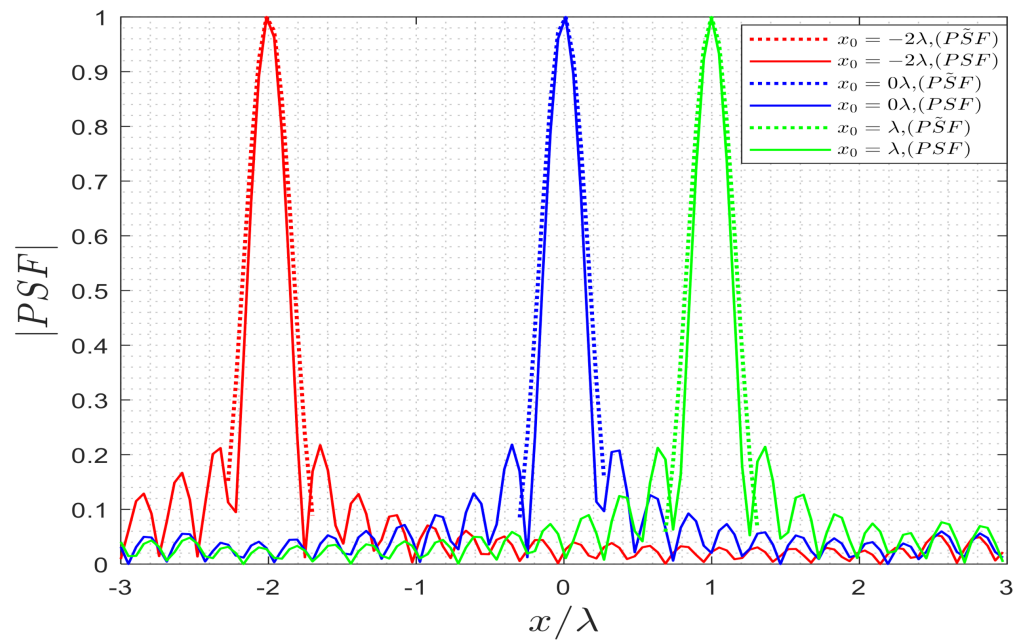

Figure 15. A comparison between the PSF (solid lines) and the $\widetilde{P S F}$ (dotted lines), with $a=3 \lambda$. 
A comparison between PSF and $\widetilde{P S F}$ is plotted in Figure 15 to check the accuracy of the resolution estimations. As can be recognized, the $\widetilde{P S F}$ approximately overlapped with the actual ones. This means that the relevant approximated $\widetilde{P S F}$ is acceptably accurate in predicting the available resolution.

In the inverse scattering problem, as can be seen in Figure 16, the width of the main lobe of PSF and $\widetilde{P S F}$ for a point scatterer is equal to $\lambda / 4$; thus, it can be defined that the minimum distance between two-point scatterers should be $\lambda / 4$ to achieve a resolvable resolution. Then, the image can be made by the combination of both PSFs of two-point scatterers. To this end, we consider two-point scatterers located in $x_{0}=-\lambda / 4$ and $x_{0}=0 \lambda$ to show the Rayleigh criterion (see Figure 16). As can be observed, the intersection point of the $\widetilde{P S F}$ (dotted black line) is approximately close to the intersection point of the PSFs (dashed black line).

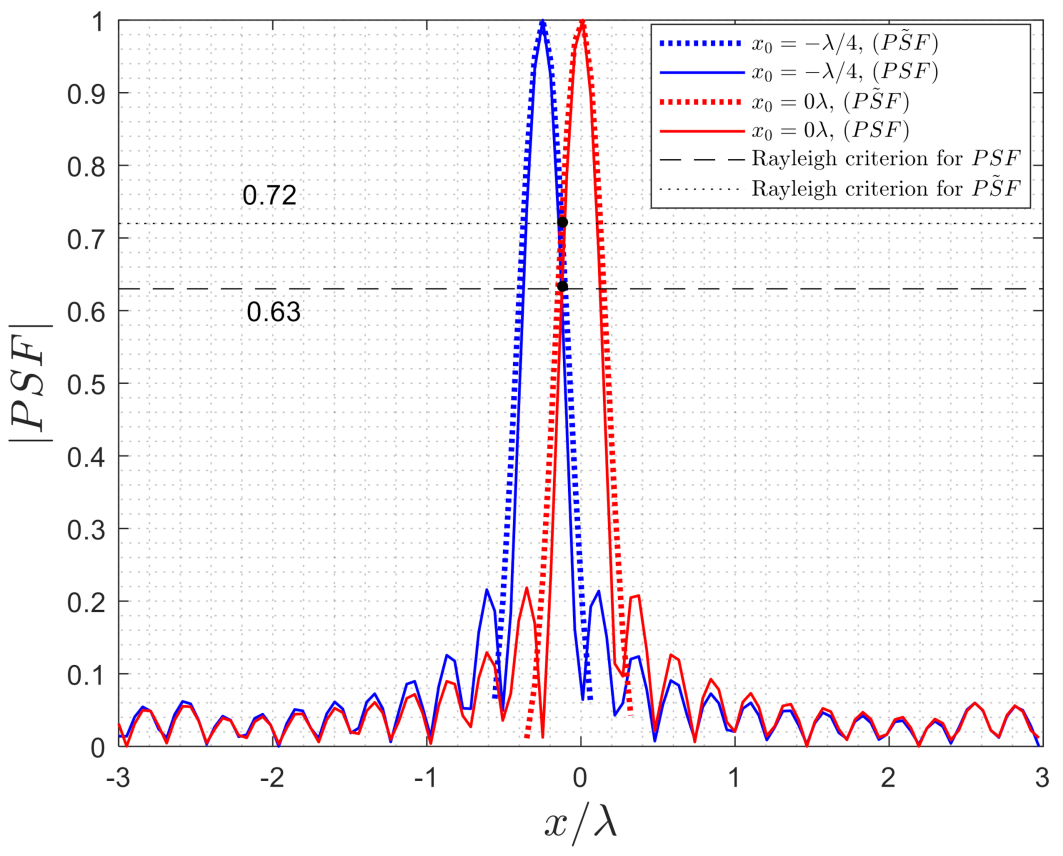

Figure 16. The Rayleigh criterion for PSF and $\widetilde{P S F}$.

\subsection{Two Strips Case}

The same analysis as Section 3.1 is here extended to address the case of two parallel stripes along the x-axis, as shown in Figure 17. We suppose two strips are located at $I_{1}=[-a, a]$ and $I_{2}=[-a, a]$, respectively, and $d=2 b$ is the distance between them. We will show the influence of the PSF of strip 1 on the other strip.

The relevant operator is defined as follows

$$
E_{T}\left(\theta_{s}, \theta_{i}\right)=\left[e^{-j \beta b\left(\sin \theta_{s}-\sin \theta_{i}\right)} \mathcal{T}_{1 s} \quad e^{j \beta b\left(\sin \theta_{s}-\sin \theta_{i}\right)} \mathcal{T}_{1 s}\right]\left[\begin{array}{l}
\chi_{1}\left(x_{1}\right) \\
\chi_{2}\left(x_{2}\right)
\end{array}\right]=\mathcal{T}_{2 s} \chi
$$

while the adjoint operator $\mathcal{T}_{2 s}^{+}$of $\mathcal{T}_{2 s}$ is given by

$$
\mathcal{T}_{2 s}^{+}=\left[\begin{array}{cc}
\mathcal{T}_{1 s}^{+} & e^{j \beta b\left(\sin \theta_{s}-\sin \theta_{i}\right)} \\
\mathcal{T}_{1 s}^{+} & e^{-j \beta b\left(\sin \theta_{s}-\sin \theta_{i}\right)}
\end{array}\right] E_{T}
$$




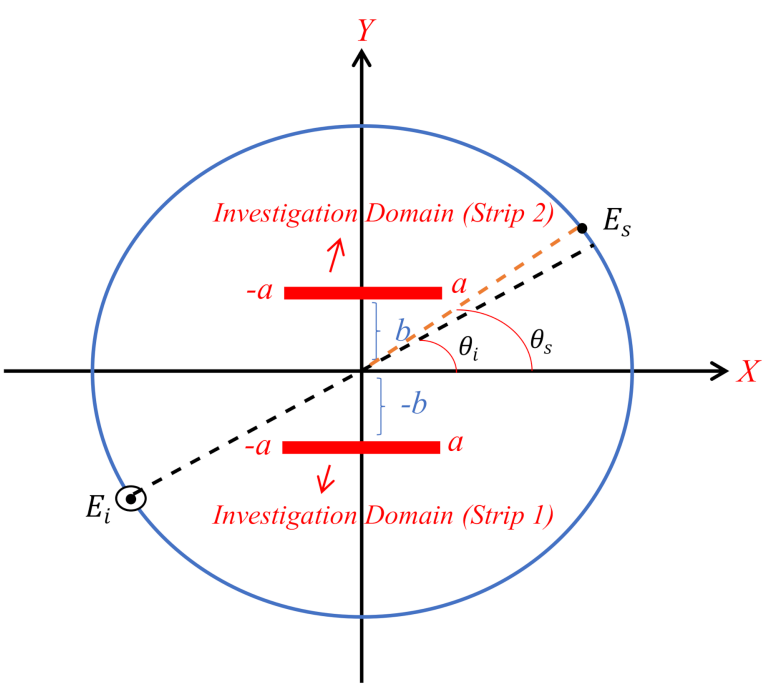

Figure 17. The geometry of the two strips case.

We provide two numerical simulations to show the PSF technique's capability to estimate the resolution and to confirm the analytical predictions. Let us consider the geometry as shown in Figure 17, in which $a$ is equal to $3 \lambda$ and the $b=\lambda$.

The first numerical simulation refers to the influence of the actual PSF of strip 1 on strip 2. As shown in Figure 18, three different source points are located at $x_{0}=-2 \lambda, 0$, and $\lambda$. As can be observed, if the location of the point scatterer changes, the width of the main lobe does not change, which means the resolution remains constant; however, the height of the PSF can vary. Furthermore, the PSF of strip 1 cannot affect strip 2.

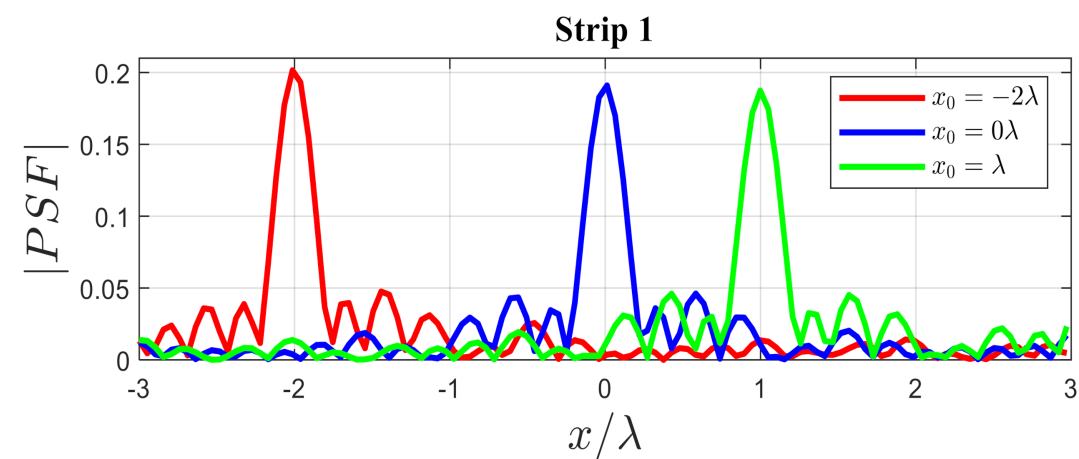

The Influence of Strip 1 on Strip 2

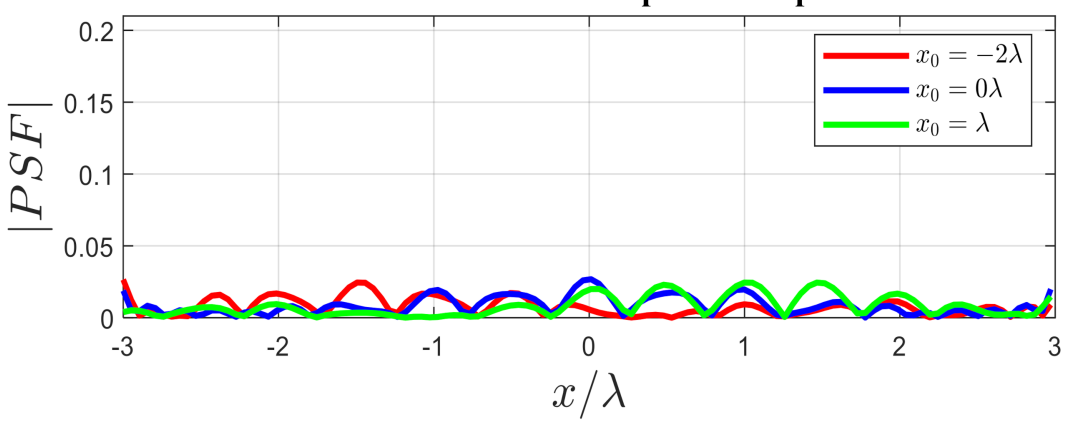

Figure 18. The influence of PSF of strip 1 on strip 2.

The second simulation concerns, again, the comparison of the results of both the PSF and $\widetilde{P S F}$ for various positions of $x_{0}$. As demonstrated in Figure 19, the main lobes of $\widetilde{P S F}$ and the PSF are approximately overlapped. 


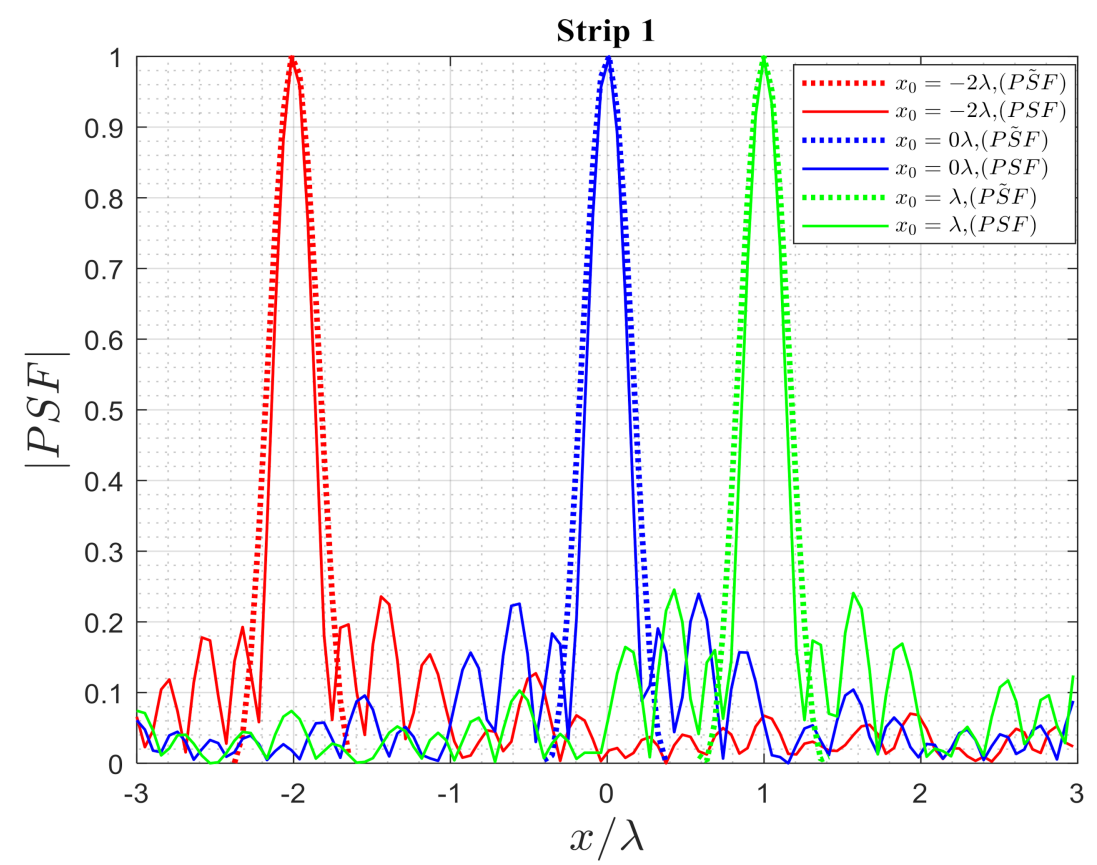

Figure 19. Comparison between the PSF (solid lines) and the $\widetilde{P S F}$ (dotted lines).

\subsection{Cross-Strips Case}

The subsection is concerned with the connected cross-strip case, as illustrated in Figure 20.

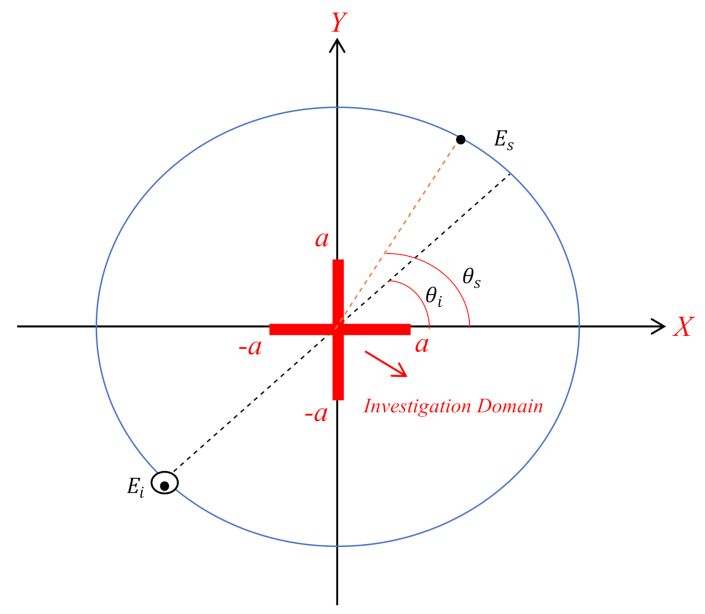

Figure 20. The geometry of the problem.

Recalling Equation (27), the far-field scattered by the horizontal strip and the vertical one is provided, respectively, by

$$
E_{s h}\left(\theta_{s}, \theta_{i}\right)=\int_{-a}^{a} \chi_{x}(x) e^{j \beta x\left(\cos \theta_{s}-\cos \theta_{i}\right)} d x=\mathcal{T}_{x}\left(\chi_{x}(x)\right)
$$

and

$$
E_{s v}\left(\theta_{s}, \theta_{i}\right)=\int_{-a}^{a} \chi_{y}(y) e^{j \beta y\left(\sin \theta_{s}-\sin \theta_{i}\right)} d y=\mathcal{T}_{y}\left(\chi_{y}(y)\right)
$$

Then the operator (34) can be considered

$$
E_{s}\left(\theta_{s}, \theta_{i}\right)=\left[\begin{array}{ll}
E_{s h}\left(\theta_{s}, \theta_{i}\right) & E_{s v}\left(\theta_{s}, \theta_{i}\right)
\end{array}\right]=\left[\begin{array}{ll}
\mathcal{T}_{x} & \mathcal{T}_{y}
\end{array}\right]\left[\begin{array}{l}
\chi_{x}(x) \\
\chi_{y}(y)
\end{array}\right]=\mathcal{T}_{x y} \chi
$$


whereas the PSF and $\widetilde{P S F}$ are the same as in Section 3.1.

The influence of the PSF of the horizontal strip on the other one is plotted in Figure 21. All of the simulation parameters are the same as the previous subsections. As can be seen, the PSF of $x_{0}=0 \lambda$ can equally affect both strips. On the other hand, the PSF of the other point scatterer cannot influence the vertical strip. We observe again that by changing the position of the point scatterer, the width of the PSF does not change, but the amplitude varies.
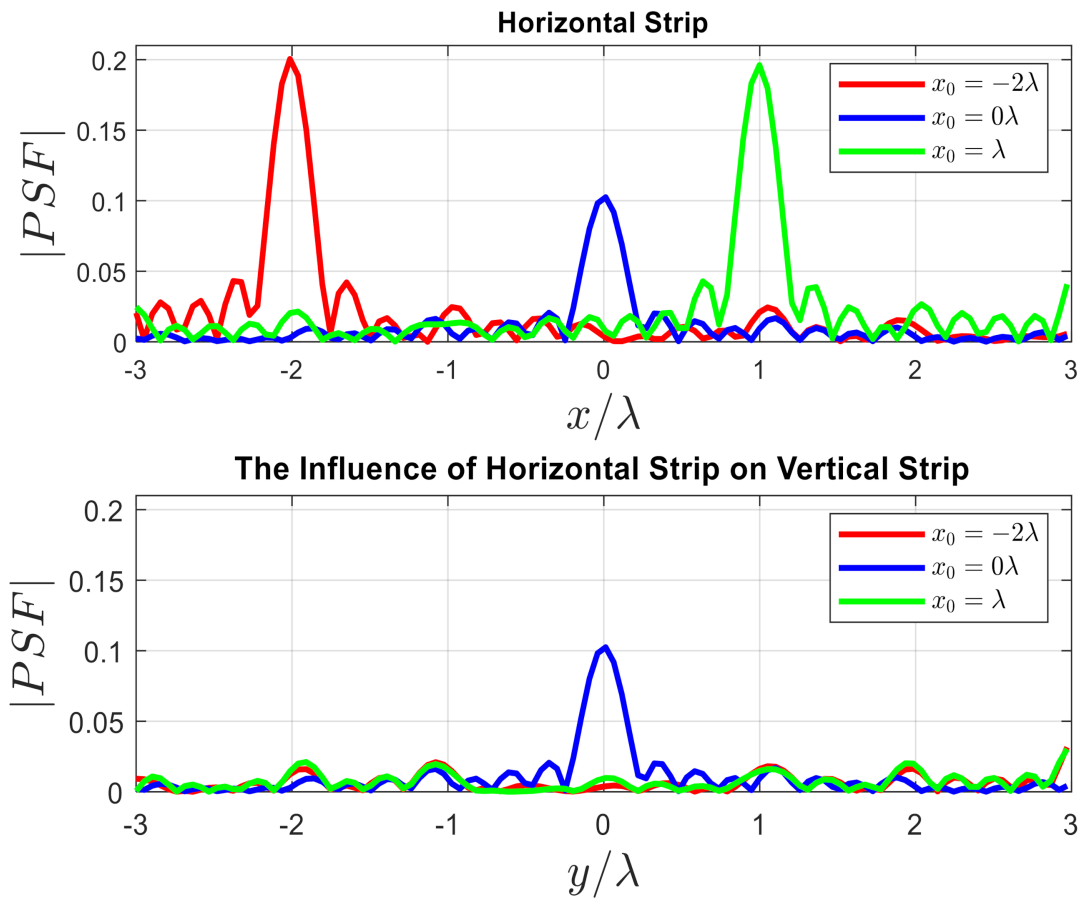

Figure 21. The influence of the PSF of the horizontal strip on the vertical strip.

A comparison between both PSF and $\widetilde{P S F}$ is shown in Figure 22, showing that the $\widetilde{P S F}$ for different point scatterers can be a good approximation of the PSF.

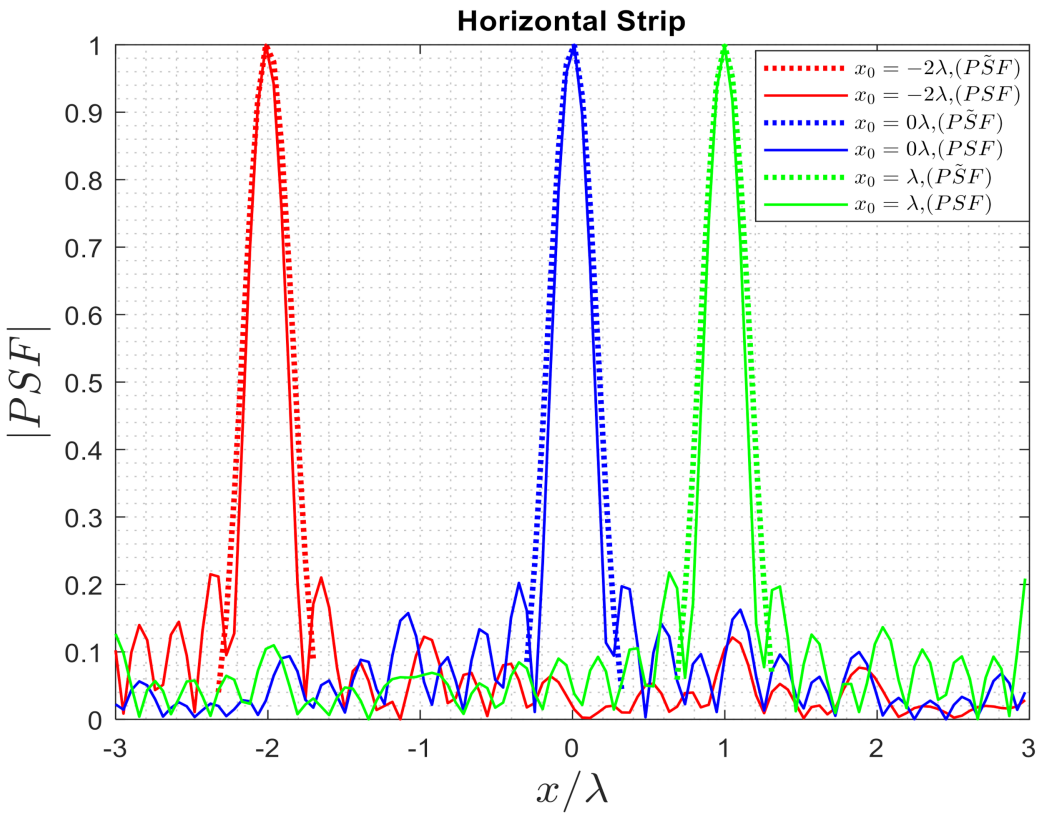

Figure 22. Comparison between the PSF and the $\widetilde{P S F}$. 
Figure 23 shows that the intersection point of $\widetilde{P S F}$ (dotted black line) is approximately close to the intersection point of PSF (dashed black line).

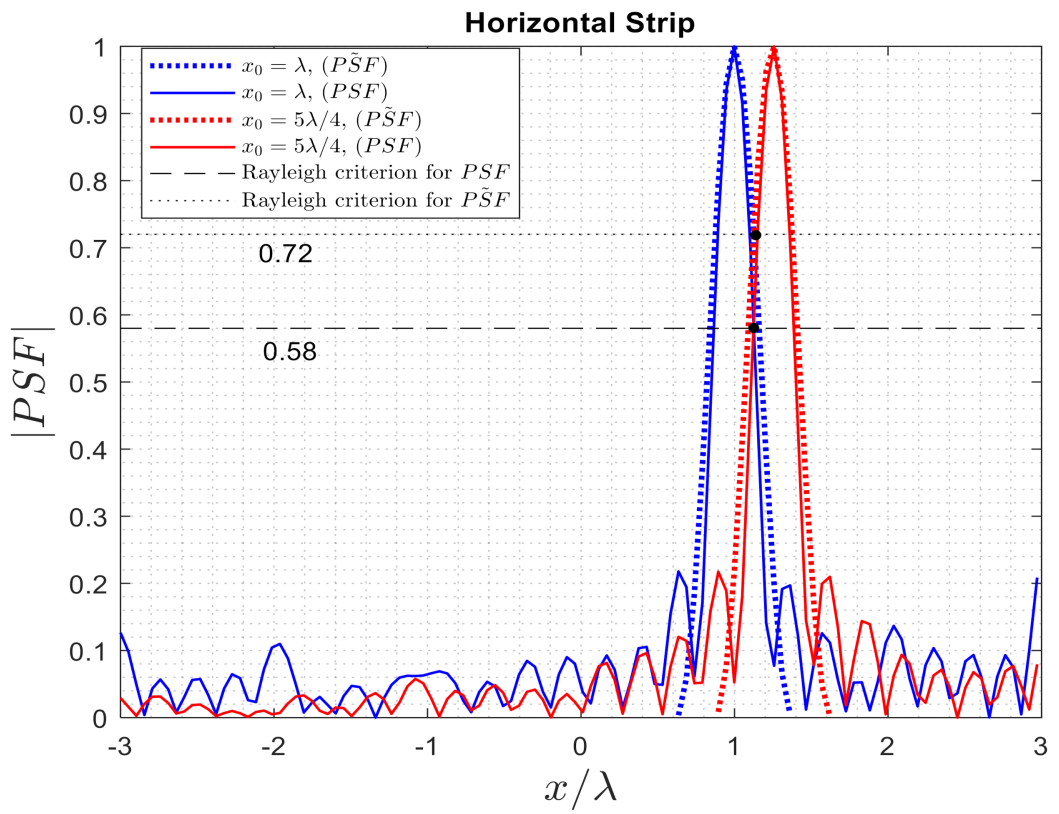

Figure 23. The Rayleigh criterion for PSF and $\widetilde{P S F}$.

The practical relevance of the above discussions can be shown by the numerical reconstruction of a cross dielectric strip with $\chi=1, b=\lambda / 10$ and $a=3 \lambda$, as illustrated in Figure 24. Then, we suppose that two cracks (free space condition, $\chi=0$ ) exist in each strip (denoted by the red regions): along the horizontal strip, the length of each crack and the distance between them is $\lambda / 4$; along the vertical strip, the length of each crack and the distance between them is $\lambda / 6$.

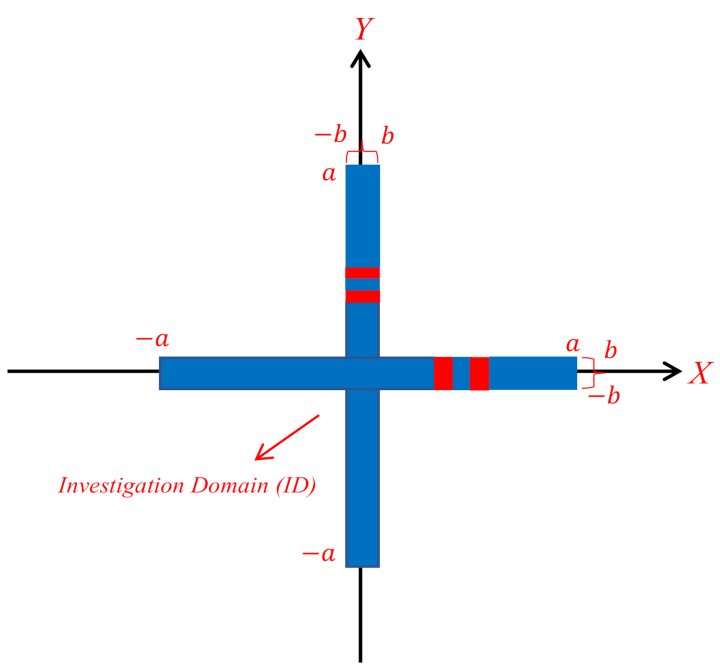

Figure 24. The geometry of the dielectric cross strip with cracks.

Figure 25 illustrates the reconstructions by inversion (34) by the TSVD algorithm for different numbers of plane waves. We expect to confirm the $\lambda / 4$ resolution limit and to show that only four plane waves are enough to achieve the best reconstructions [26]. As can be observed, by considering only four incident plane waves (red line) along the horizontal strip, it can be distinguished that two cracks are present; oppositely, it is impossible to differentiate between two cracks in the vertical strip, even with the maximum number 
of incident plane waves. Therefore the resolution analysis is important for the reliable reconstruction of defects in dielectric objects in inverse scattering problems.
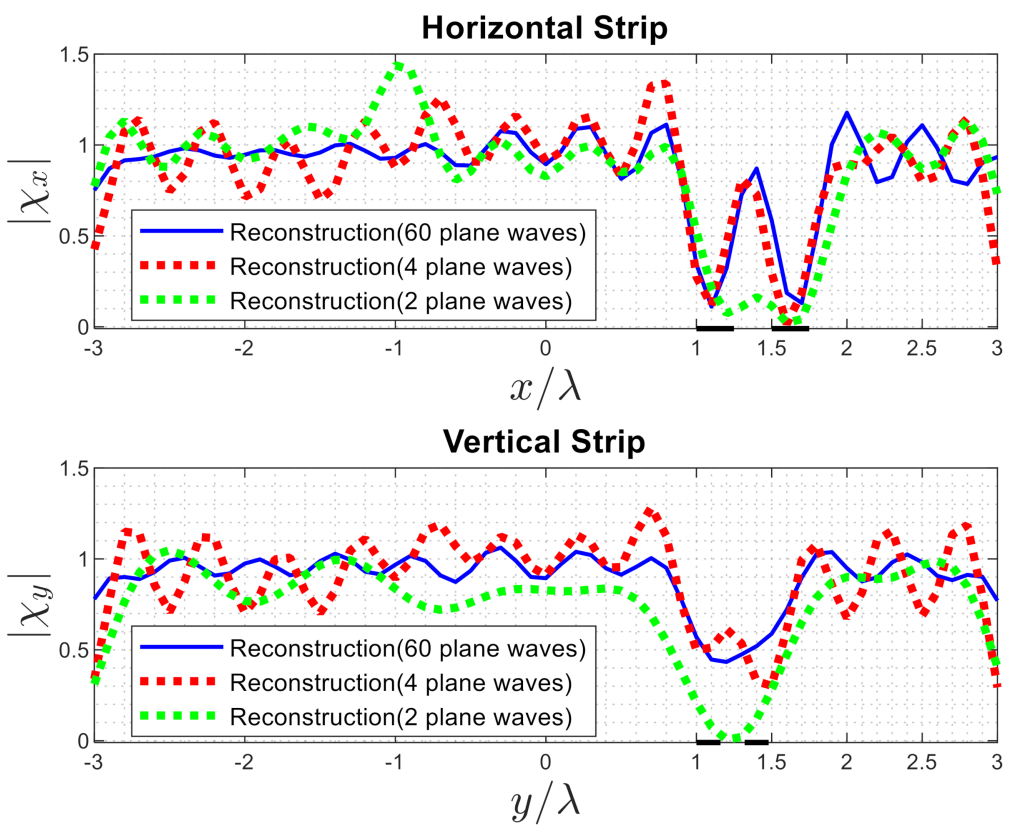

Figure 25. The reconstructions for different numbers of plane waves.

\section{Conclusions}

The goal of this paper is more methodological than practical: it aims at investigating the role of the PSF in both the inverse source and the inverse scattering problems. Fundamentally, the PSF provides the reconstruction of a point-like source/object. Accordingly, it can be useful in array diagnostics, for instance, since it provides the minimum distance between its elements for a reliable excitation reconstruction, or in non-destructive testing of dielectric objects since it provides the minimum dimension of a defect that can be reconstructed by sensing the object via electromagnetic waves, as in microwave tomography. We have investigated the PSF's role in the linear electromagnetic inverse for both source and scattering problems to estimate the achievable resolution. The PSF can be computed through the numerical solution of the pertinent inverse problem. Moreover, it depends on the source/object geometry; then, it would be very fruitful to be able to find a closed-form, though approximate, function. Since, in principle, it depends on the unknown function geometry domain, in this paper, we have chosen to start with simple strip geometries made of a collection of linear domains. In this way, some closed-form results in inverse problems can be achieved, such as the NDF, and its connection with the PSF can be established. However, since the exact evaluation of the PSF can be performed only numerically in most circumstances, an approximate analytical evaluation has been introduced, and its accuracy is assessed against the actual PSF for each geometry by numerical simulations. The Rayleigh criterion has also been checked for both PSFs to confirm the analytical discussion. When the observation domain scans the full angle, space invariant PSF is achieved, which means that the maximum resolution of the reconstruction of point-like sources/objects is achieved independently on their locations within the investigation domain. In particular, for the considered geometries made of parallel or cross strips, a $\lambda / 2$ and $\lambda / 4$ resolution is appreciated for the inverse source and scattering problems, respectively. The presented discussion, allowing the connection of the PSF analysis to the NDF and to the resolution in linear electromagnetic inverse problems in simple geometries, provides the foundation to the general 2D case. The relevance of the approach to antenna diagnostics and microwave tomography of dielectric objects has been shown by two numerical examples. 
Author Contributions: Conceptualization, G.L. and R.P.; methodology, G.L. and R.P.; software, E.A.S.; validation, E.A.S. and G.L.; formal analysis, E.A.S. and G.L.; investigation, E.A.S. and G.L.; resources, E.A.S.; data curation, E.A.S.; writing-original draft preparation, E.A.S.; writing-review and editing, G.L.; visualization, G.L.; supervision, R.P.; project administration, G.L.; funding acquisition, R.P. All authors have read and agreed to the published version of the manuscript.

Funding: This research received no external funding.

Data Availability Statement: Data supporting reported results are generated during the study.

Conflicts of Interest: The authors declare no conflict of interest.

\section{References}

1. Tarantola, A. Inverse Problem Theory and Methods for Model Parameter Estimation; SIAM: Philadelphia, PA, USA, 2005.

2. Sever, A. An inverse problem approach to pattern recognition in industry. Appl. Comput. Inform. 2015, 11, 1-12. [CrossRef]

3. Leone, G. Source geometry optimization for hemispherical radiation pattern coverage. IEEE Trans. Antennas Propag. 2016, 64, 2033-2038. [CrossRef]

4. Maisto, M.A.; Moretta, R.; Solimene, R.; Pierri, R. Phaseless arrays diagnostic by phaselift in near zone: Numerical experiments. In Proceedings of the 2017 Progress in Electromagnetics Research Symposium-Spring (PIERS), St. Petersburg, Russia, 22-25 May 2017.

5. Dell'Aversano, A.; Leone, G.; Ciaramaglia, F.; Solimene, R. A Strategy for Reconstructing Simple Shapes From Undersampled Backscattered Data. IEEE Geosci. Remote Sens. Lett. 2016, 13, 1757-1761. [CrossRef]

6. Chen, X. Computational Methods for Electromagnetic Inverse Scattering; Wiley Online Library: Hoboken, NJ, USA, 2018.

7. Brancaccio, A.; Solimene, R. Fault detection in dielectric grid scatterers. Opt. Express 2015, 23, 8200-8215. [CrossRef]

8. Rossmann, K. Point spread-function, line spread-function, and modulation transfer function: Tools for the study of imaging systems. Radiology 1969, 93, 257-272. [CrossRef]

9. Goodman, J. Introduction to Fourier Optics; Freeman: New York, NY, USA, 2017.

10. Nakahara, H.; Haney, M.M. Point spread functions for earthquake source imaging: An interpretation based on seismic interferometry. Geophys. J. Int. 2015, 202, 54-61. [CrossRef]

11. Miller, C.R.; Routh, P.S. Resolution analysis of geophysical images: Comparison between point spread function and region of data influence measures. Geophys. Prospect. 2007, 55, 835-852. [CrossRef]

12. Javaherian, A.; Movafeghi, A.; Faghihi, R.; Yahaghi, E. An exhaustive criterion for estimating quality of images in electrical impedance tomography with application to clinical imaging. J. Vis. Commun. Image Represent. 2013, 24, 773-785. [CrossRef]

13. Dalitz, C.; Pohle-Frohlich, R.; Michalk, T. Point spread functions and deconvolution of ultrasonic images. IEEE Trans. Ultrason. Ferroelectr. Freq. Control 2015, 62, 531-544. [CrossRef]

14. Schuster, G.T.; Hu, J. Green's function for migration: Continuous recording geometry. Geophysics 2000, 65, 167-175. [CrossRef]

15. Pierri, R.; Liseno, A.; Soldovieri, F.; Solimene, R. In-depth resolution for a strip source in the Fresnel zone. J. Opt. Soc. Am. A Opt. Image Sci. Vis. 2001, 18, 352-359. [CrossRef]

16. Solimene, R.; Maisto, M.A.; Pierri, R. Information content in inverse source with symmetry and support priors. Prog. Electromagn. Res. 2018, 80, 39-54. [CrossRef]

17. Leone, G.; Munno, F.; Pierri, R. Inverse Source on Conformal Conic Geometries. IEEE Trans. Antennas Propag. 2021, 69, 1596-1609. [CrossRef]

18. Maisto, M.A.; Solimene, R.; Pierri, R. Resolution limits in inverse source problem for strip currents not in Fresnel zone. JOSA $A$ 2019, 36, 826-833. [CrossRef]

19. Leone, G.; Munno, F.; Pierri, R. Radiation properties of conformal antennas: The elliptical source. Electronics $2019,8,531$. [CrossRef]

20. Pierri, R.; Liseno, A.; Solimene, R.; Tartaglione, F. In-depth resolution from multifrequency Born fields scattered by a dielectric strip in the Fresnel zone. JOSA A 2002, 19, 1234-1238. [CrossRef] [PubMed]

21. Solimene, R.; Leone, G.; Pierri, R. Resolution in two-dimensional tomographic reconstructions in the Fresnel zone from Born scattered fields. J. Opt. A Pure Appl. Opt. 2004, 6, 529. [CrossRef]

22. Slepian, D.; Pollak, H.O. Prolate spheroidal wave functions, fourier analysis and uncertainty-I. Bell Syst. Tech. J. 1961, 40, 43-63. [CrossRef]

23. Leone, G.; Maisto, M.A.; Pierri, R. Application of Inverse Source Reconstruction to Conformal Antennas Synthesis. IEEE Trans. Antennas Propag. 2018, 66, 1436-1445. [CrossRef]

24. Bertero, M.; Boccacci, P. Introduction to Inverse Problems in Imaging; IOP Publishing: Bristol, UK, 1998.

25. Solimene, R.; Maisto, M.A.; Pierri, R. Sampling approach for singular system computation of a radiation operator. J. Opt. Soc. Am. A Opt. Image Sci. Vis. 2019, 36, 353-361. [CrossRef]

26. Sekehravani, E.A.; Leone, G.; Pierri, R. NDF of Scattered Fields for Strip Geometries. Electronics 2021, 10, 202. [CrossRef] 